Supporting Information

\title{
Cephalopod-Inspired Chromotropic Ionic Skin with Rapidly Visual Sensing Capabilities to Multiple Stimuli
}

Yunpeng Wang, Xianfei Cao, Jie Cheng, Bowen Yao, Yusen Zhao, Suli Wu, Benzhi Ju, Shufen Zhang, Ximin He*, and Wenbin Niu*

Y. Wang, X. Cao, J. Cheng, Prof. S. Wu, Prof. B. Ju, Prof. S. Zhang, Prof. W. Niu State Key Laboratory of Fine Chemicals, Dalian University of Technology, West Campus, 2 Linggong Rd., Dalian 116024, China.

E-mail: niuwb@dlut.edu.cn

B. Yao, Y. Zhao, Prof. X. He

Department of Materials Science and Engineering, University of California, Los Angeles, CA 90095, USA

E-mail: ximinhe@ucla.edu 

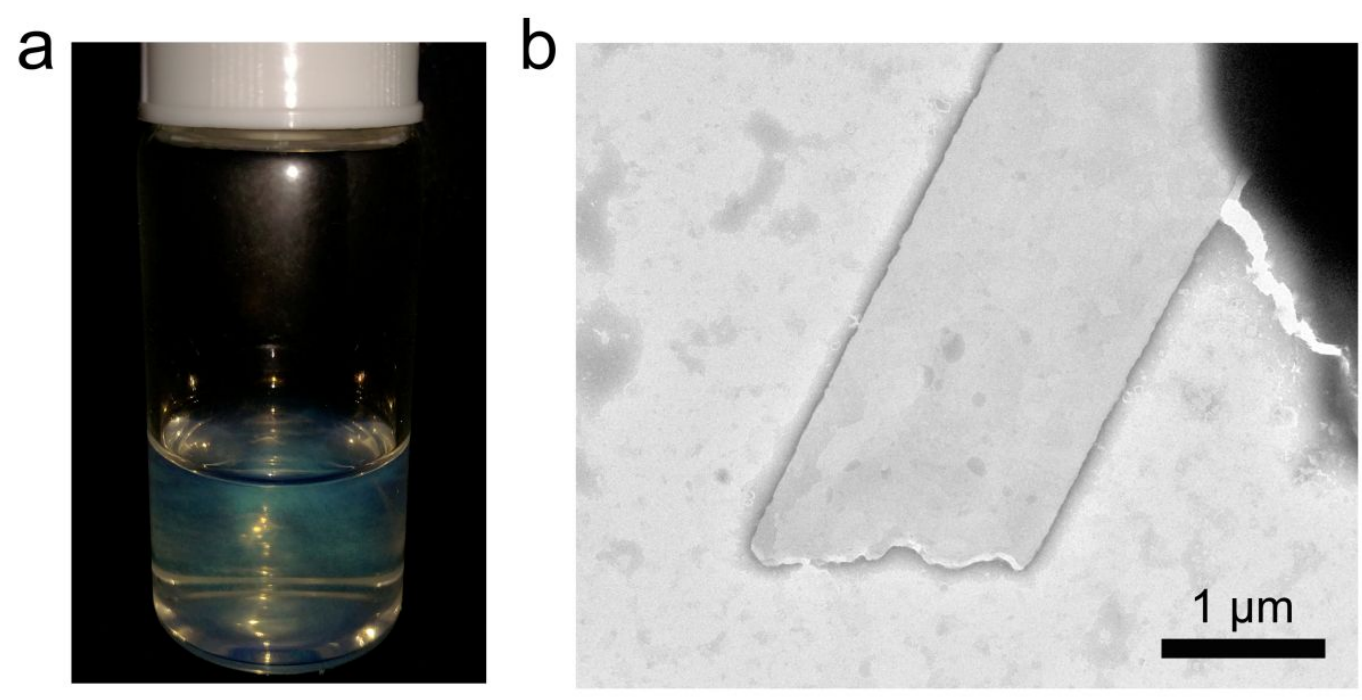

Figure S1. Properties of $\mathrm{C}_{12} \mathrm{DMAO}$ aqueous solution. (a) Structural color of $\mathrm{C}_{12}$ DMAO solution, in which $\mathrm{C}_{12}$ DMAO is $30 \mathrm{mg}, n$-hexanol is $53 \mathrm{mg}$, water is $5 \mathrm{~mL}$, and the ambient temperature is $15^{\circ} \mathrm{C}$. (b) TEM image of $\mathrm{C}_{12}$ DMAO bilayers. 


\section{a}
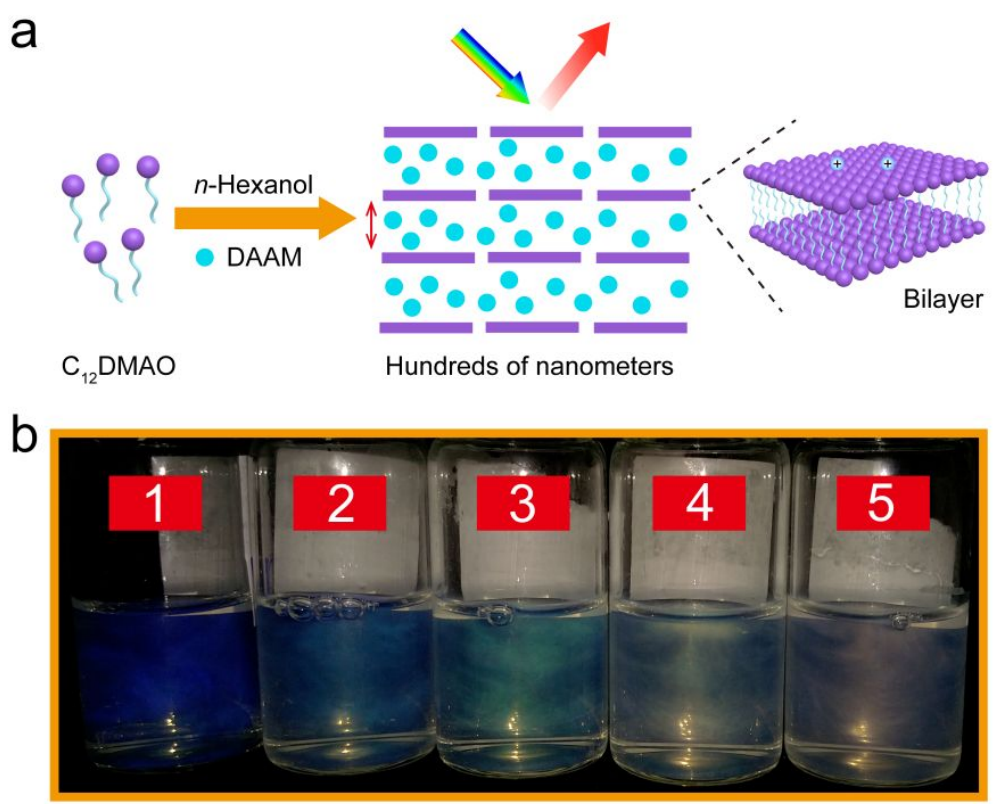

Figure S2. $\mathrm{C}_{12} \mathrm{DMAO}$ solution containing the polymerizable monomer DAAM. (a)

Schematic diagram of the lamellar liquid crystals composed of $\mathrm{C}_{12} \mathrm{DMAO}$ molecules self-assembled in water containing DAAM. (b) Digital images of solutions with different structural colors.

As shown in Figure $\mathrm{S} 2 \mathrm{~b}$, with the decrease of $\mathrm{C}_{12} \mathrm{DMAO}$ content, the distance between the bilayers gradually increased, leading to the gradual increase of Bragg diffraction peak of solution and the red shift of structure color. The corresponding specific components are shown in Table S1. The appearance of structural colors indicates that $\mathrm{C}_{12} \mathrm{DMAO}$ molecules self-assemble into bilayers structure in water and form Bragg stack due to electrostatic repulsion. ${ }^{\mathrm{S} 1}$ And the introduction of DAAM does not destroy this structure, which makes the preparation of photonic hydrogels possible. 
Table S1. The components of solutions with different structural colors in Figure S2b.

\begin{tabular}{ccccc}
\hline & $\begin{array}{c}\mathrm{C}_{12} \text { DMAO } \\
(\mathrm{mg})\end{array}$ & $\begin{array}{c}n \text {-Hexanol } \\
(\mathrm{mg})\end{array}$ & $\begin{array}{c}\text { DAAM } \\
(\mathrm{g})\end{array}$ & $\begin{array}{c}\mathrm{H}_{2} \mathrm{O} \\
(\mathrm{mL})\end{array}$ \\
\hline 1 & 39 & 67 & 0.50 & 5 \\
2 & 36 & 66 & 0.50 & 5 \\
3 & 33 & 64 & 0.45 & 5 \\
4 & 30 & 63 & 0.45 & 5 \\
5 & 27 & 60 & 0.45 & 5 \\
\hline
\end{tabular}


Table S2. The specific components of the precursors for preparing ICPH.

\begin{tabular}{cccccccc}
\hline $\begin{array}{c}\lambda \\
(\mathrm{nm})\end{array}$ & $\begin{array}{c}\mathrm{C}_{12} \text { DMAO } \\
(\mathrm{mg})\end{array}$ & $\begin{array}{c}n \text {-Hexanol } \\
(\mathrm{mg})\end{array}$ & $\begin{array}{c}\text { DAAM } \\
(\mathrm{g})\end{array}$ & $\begin{array}{c}\text { AAm } \\
(\mathrm{g})\end{array}$ & $\begin{array}{c}\text { MBAA } \\
(\mathrm{mg})\end{array}$ & $\begin{array}{c}\text { TPO } \\
(\mathrm{mg})\end{array}$ & $\begin{array}{c}\text { water } \\
(\mathrm{mL})\end{array}$ \\
\hline 650 & 60 & 85 & 0.5 & 0.25 & 2 & 2.5 & 5 \\
610 & 60 & 85 & 0.5 & 0.25 & 4 & 2.5 & 5 \\
552 & 60 & 85 & 0.5 & 0.25 & 6 & 2.5 & 5 \\
495 & 60 & 85 & 0.5 & 0.25 & 8 & 2.5 & 5 \\
465 & 60 & 85 & 0.5 & 0.25 & 12 & 2.5 & 5 \\
\hline
\end{tabular}

By introducing polymerizable monomers into $\mathrm{C}_{12} \mathrm{DMAO}$ solution, we successfully fixed lamellar liquid crystals in the hydrogel network to obtain photonic hydrogels with structural colors. The polymerized diacetone acrylamide (PDAAM) is hydrophobic, so it is necessary to introduce the hydrophilic monomer acrylamide into the solution to form hydrogel matrix by UV curing. 


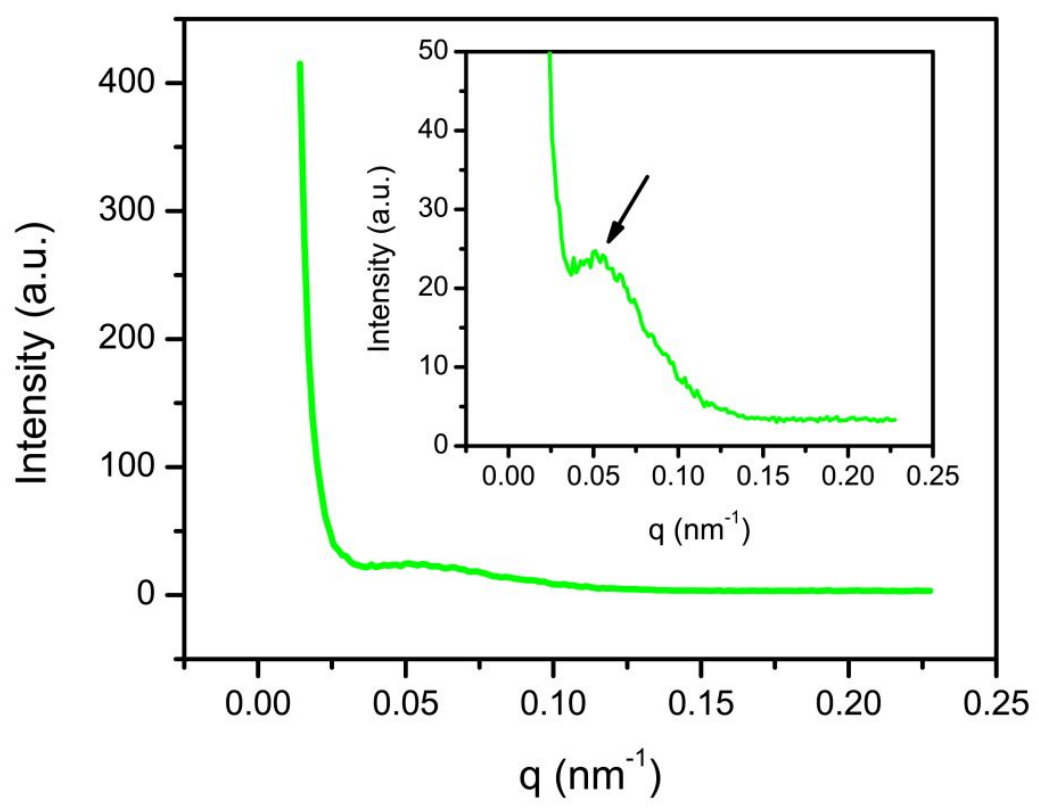

Figure S3. The SAXS profile of ICPH, inset is a partial enlarged view. 


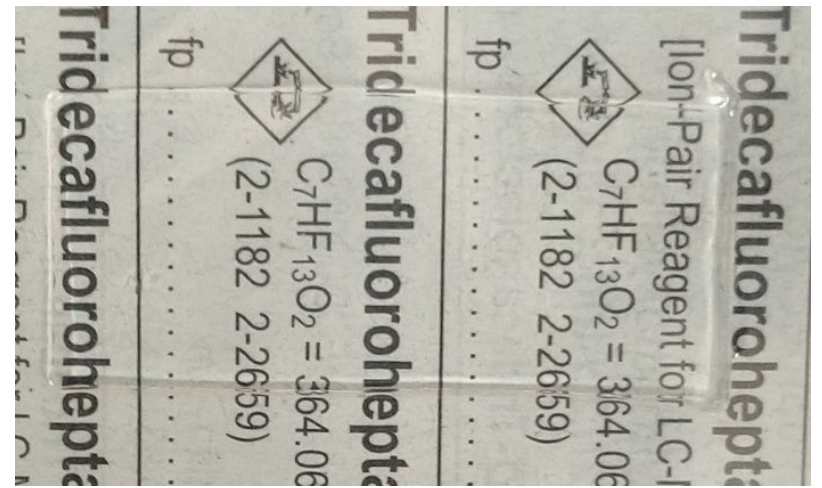

Figure S4. Digital image of $\mathrm{C}_{12} \mathrm{DMAO}$ photonic hydrogel.

The unswollen $\mathrm{C}_{12}$ DMAO photonic hydrogel is in a transparent state. After swelling in deionized water, the distance between two bilayers increases, leading to the appearance and red shift of structural color. Therefore, the structural color can be precisely controlled by adjusting the crosslinker. 


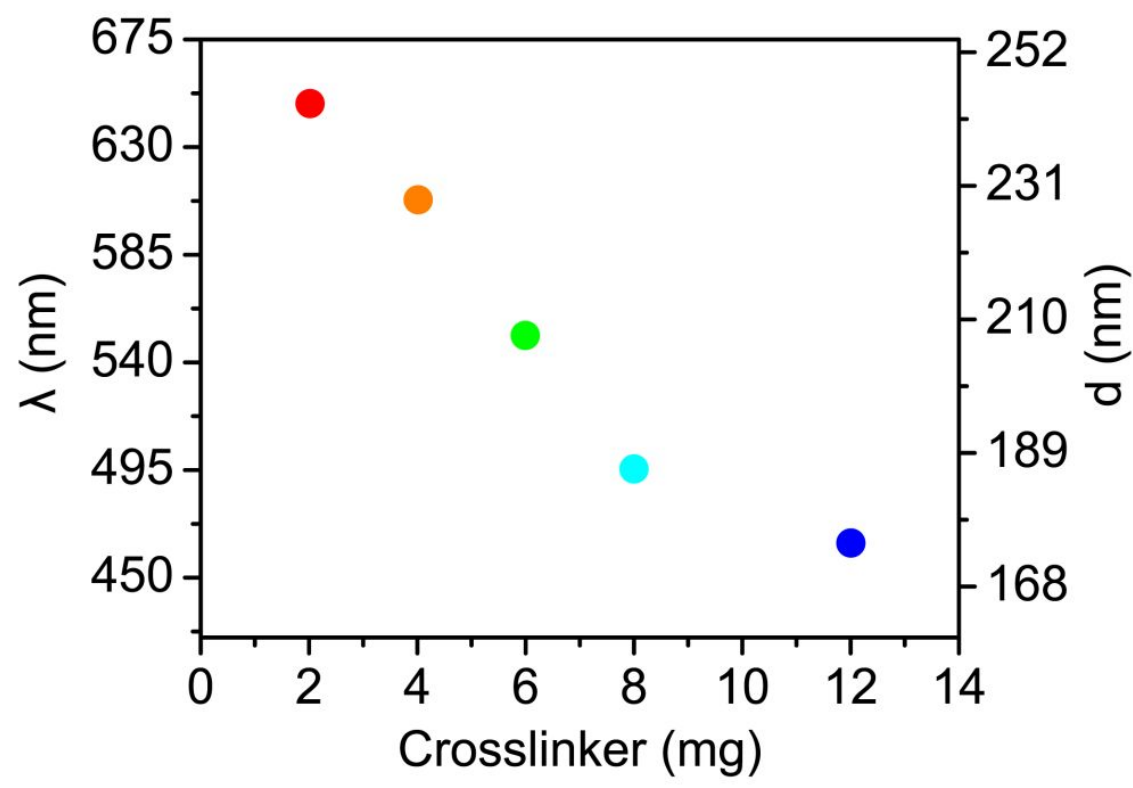

Figure S5. The maximum reflection peak wavelength and the distance $d$ between the bilayers of hydrogel prepared under different crosslinker. $d$ is calculated according to the Bragg equation, in which $n$ is the refractive index of water at 1.33 and $\theta$ is the incidence angle of $90^{\circ} . \mathrm{S} 2$ 


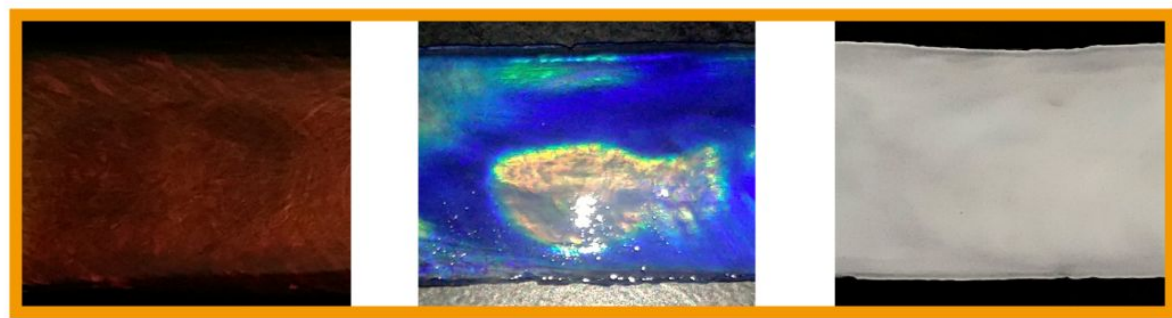

$\mathrm{NaCl}: 2 \mathrm{wt} \%$

$3 w t \%$

$10 w t \%$

Figure S6. The structural color of red hydrogels in $\mathrm{NaCl}$ solutions of different mass concentrations.

When the sample was immersed in $2 \mathrm{wt} \% \mathrm{NaCl}$ solution, the structural color was still red; however, in $3 \mathrm{wt} \% \mathrm{NaCl}$ solution, the structural color was blue shifted due to the high osmotic pressure, which caused the dehydration and volume reduction of the hydrogel. Moreover, the hydrogel will dehydrate and turn white at a higher concentration $(10 \mathrm{wt} \%)$. 

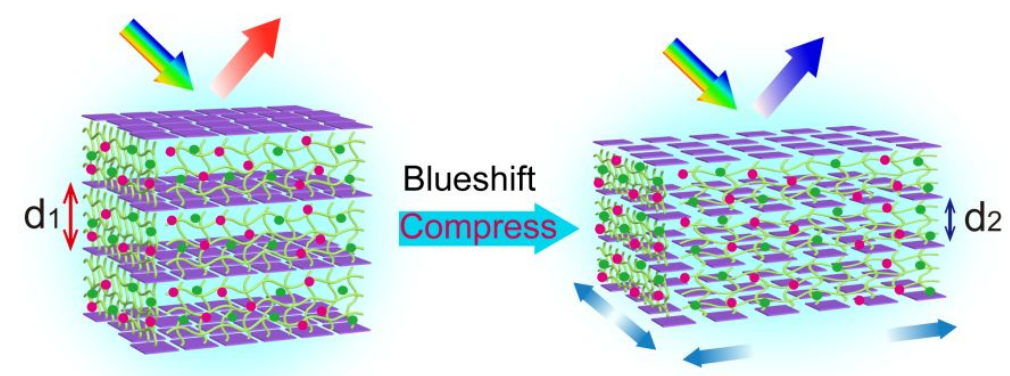

Figure S7. Mechanism illustration of the structural color blue shift behavior under compression. 


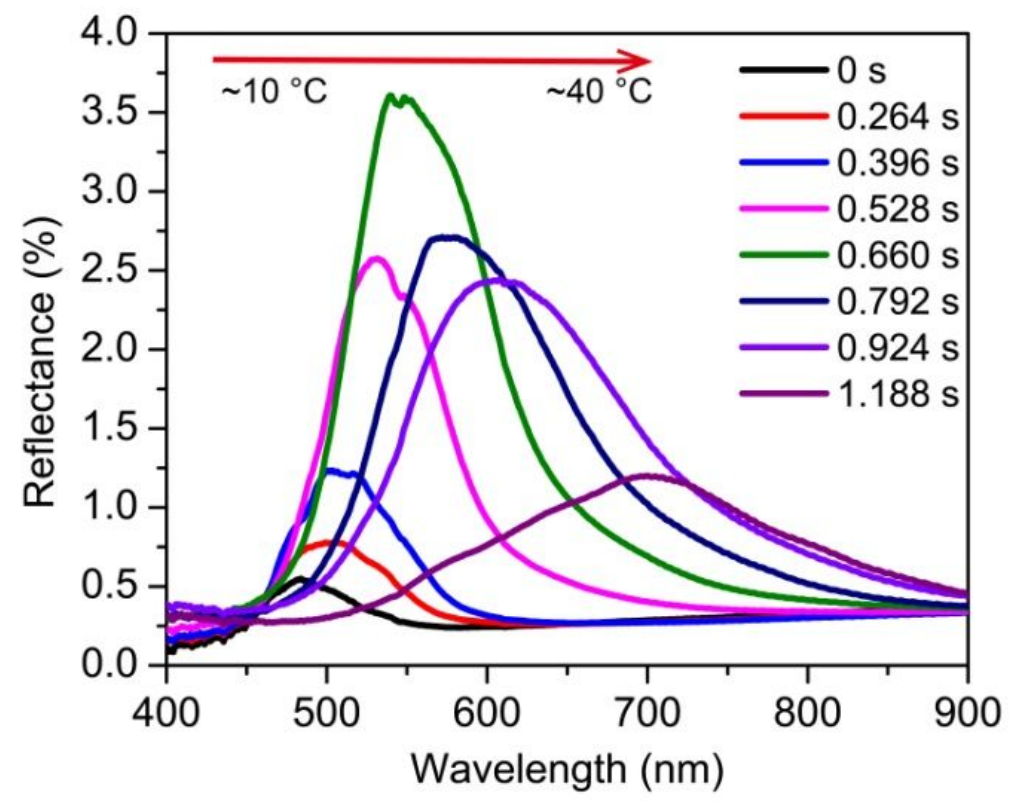

Figure S8. Continuous changes of the reflection spectrum with time from about $10{ }^{\circ} \mathrm{C}$ to $40{ }^{\circ} \mathrm{C}$. 


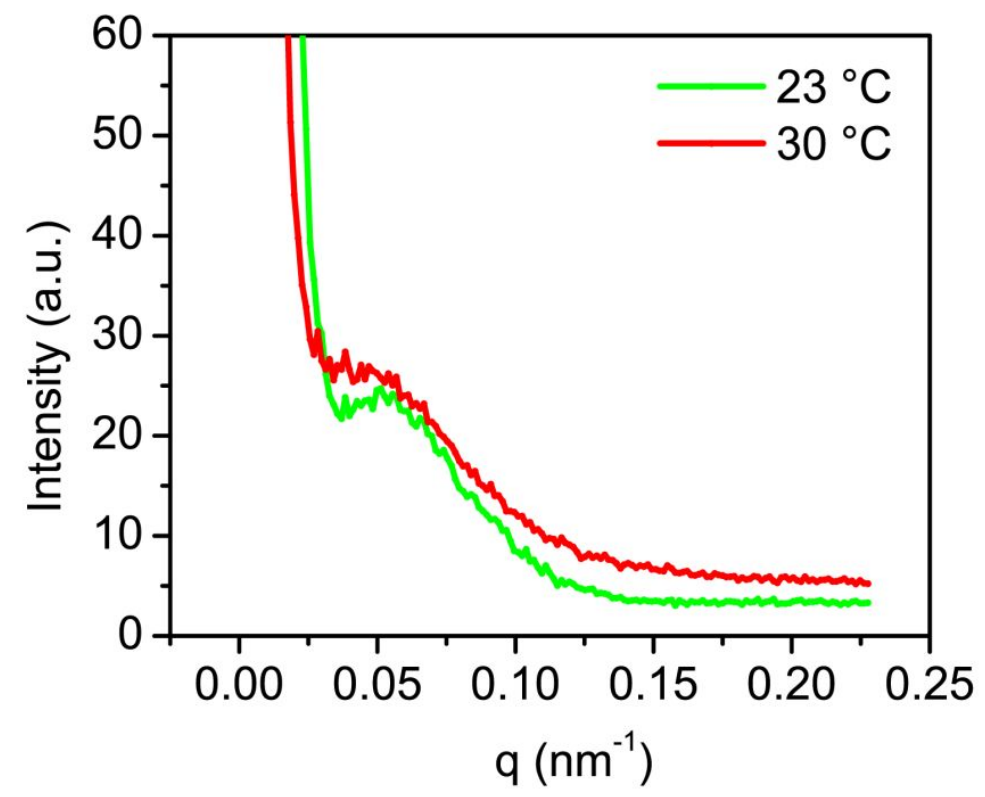

Figure S9. SAXS profiles of ICPH at different temperatures. The distance $d$ between the bilayers is given by the following relationship, ${ }^{\mathrm{S} 3} d=2 \pi / q$. Therefore, a decrease in $q$ proves the expansion of the spacing between the bilayers. 


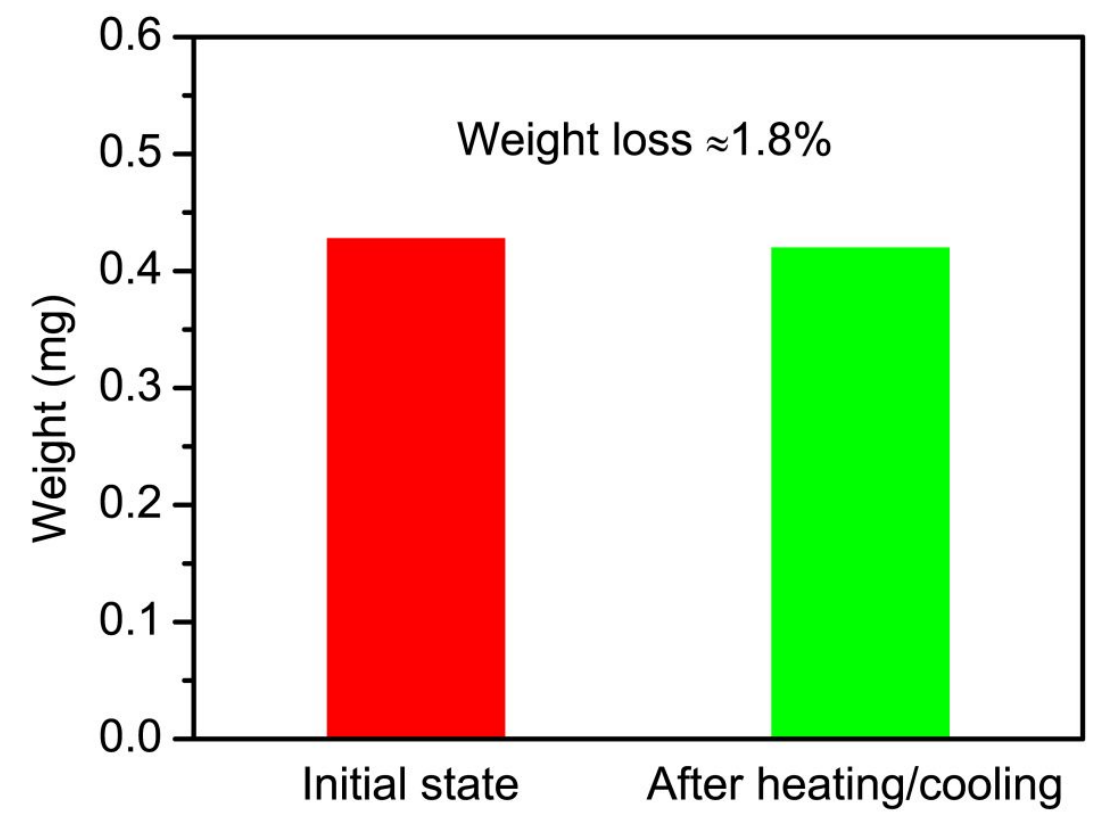

Figure S10. Weight of ICPH before and after heating/cooling in air. 


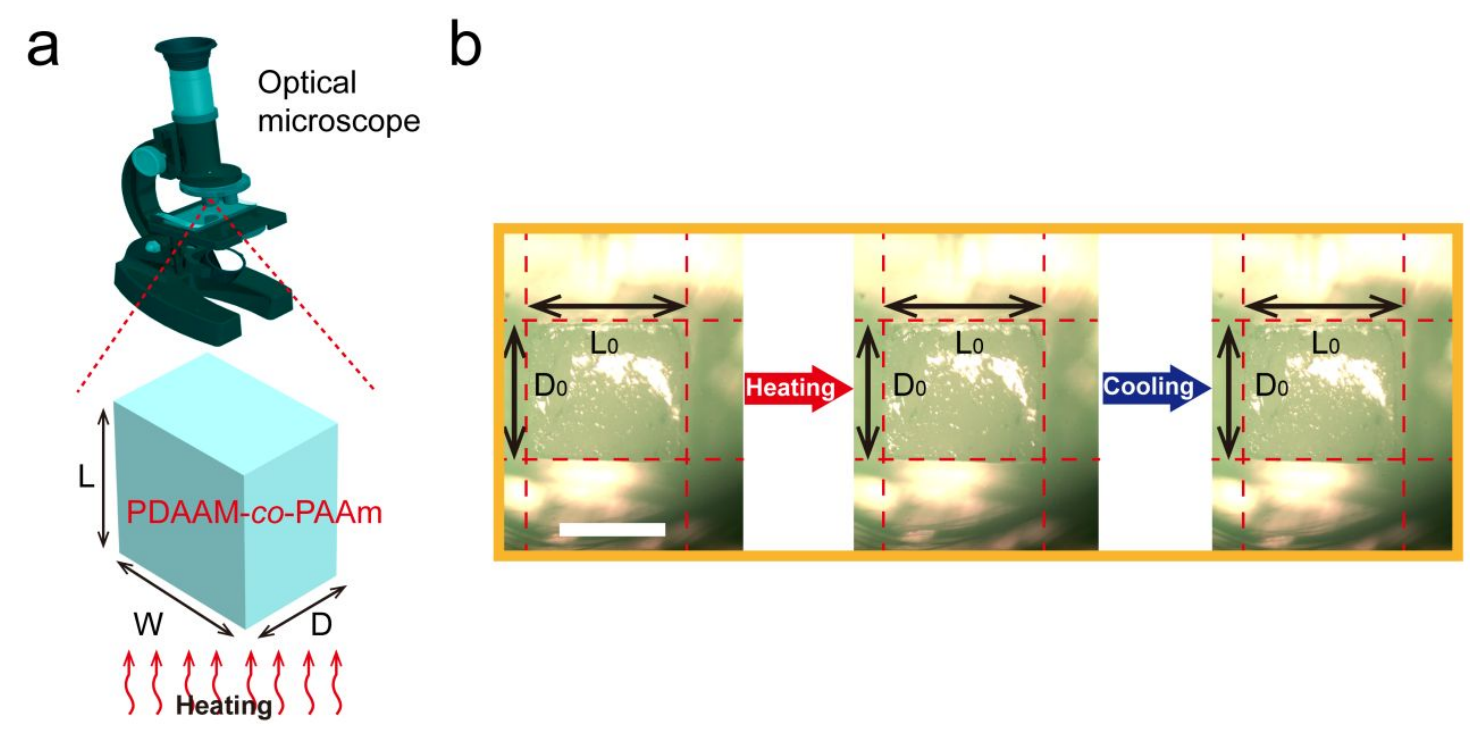

Figure S11. Change in the volume of pure PDAAM-co-PAAM hydrogel without $\mathrm{C}_{12}$ DMAO bilayers. (a) Schematic diagram of the volume change observed using an optical microscope. (b) Static images of the volume change during heating/cooling cycle $\left(20-40{ }^{\circ} \mathrm{C}\right)$. Scale bar is $1 \mathrm{~mm}$. There is no change in volume of pure PDAAM-co-PAAM hydrogel, indicating that anisotropic volume change of ICPH is caused by the charged bilayers rather than the hydrogel matrix. 
a

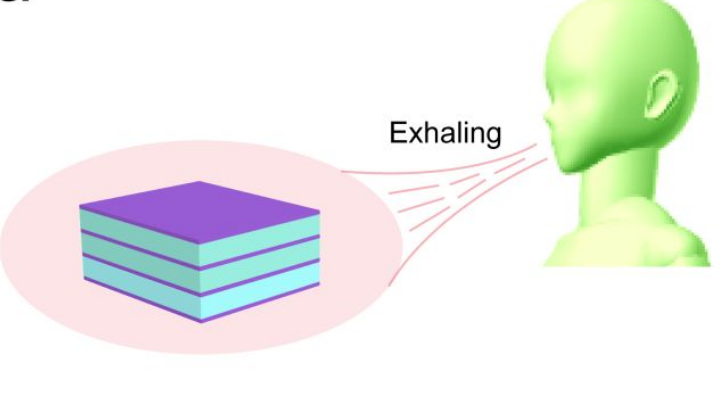

b

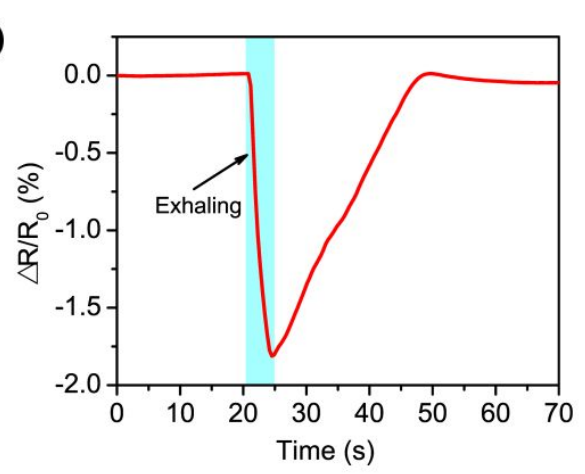

Figure S12. Response of ICPH to heat exhaled from human. (a) Schematic diagram of hydrogels sensing heat exhaled. (b) Change of relative resistance after contact heat exhaled. When the sample contacts the human exhaled breath, the relative resistance decreases rapidly, and it returns to the initial state after stopping the breath. 


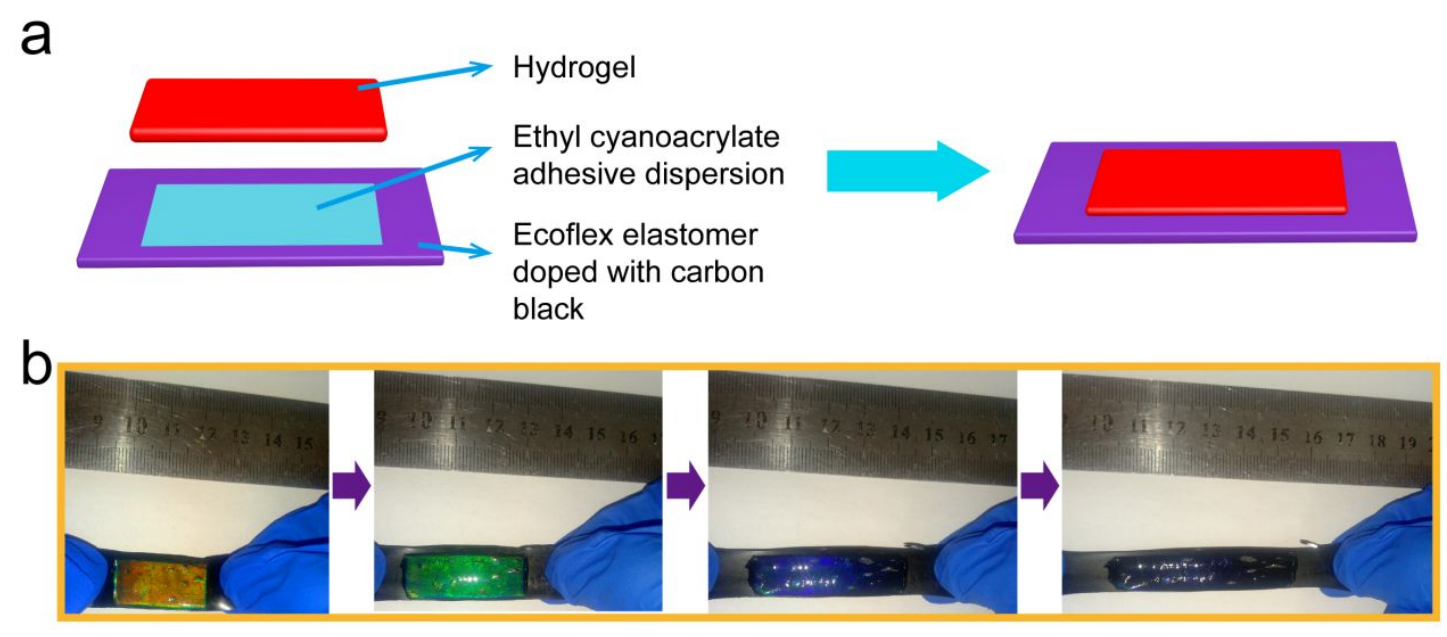

Figure S13. Integration of ionic sensory system as biomimetic CIskin. (a) Schematic diagram of hydrogel and dielectric elastomer integrated into device. (b) Digital images of the device during stretching.

In order to avoid the interference of background color on the structural color of ICPH, Ecoflex elastomers doped with carbon black were used as dielectric elastomer, and it was combined with ICPH and PAAM hydrogels by adhesive dispersion to avoid separation during tensile testing. As shown in Figure 13a, in order to ensure the extensibility of the integrated device, ethyl cyanoacrylate adhesive was dispersed in cyclohexane (mass ratio 1:5) as adhesive dispersion. Figure 13b shows that the device still has excellent tensile properties because diluted ethyl cyanoacrylate does not form a rigid resin intermediate between hydrogels and elastomer. ${ }^{\mathrm{S} 4}$ 


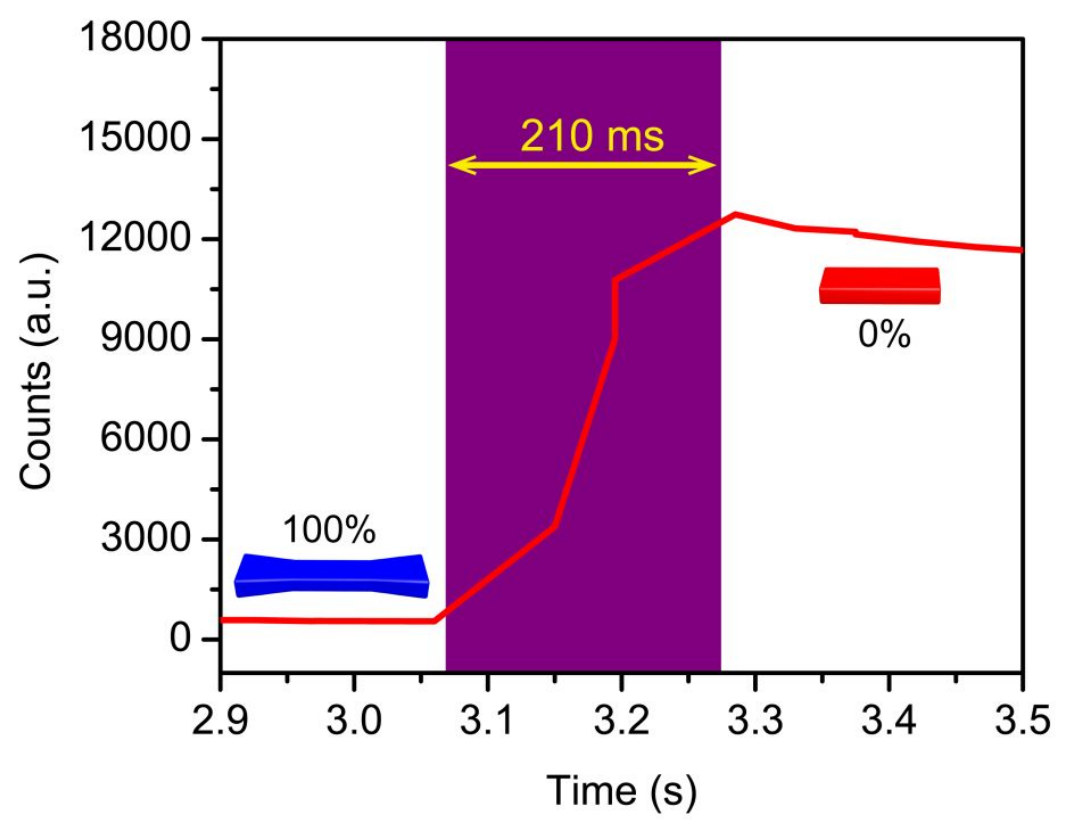

Figure S14. Response time of optical signal from $100 \%$ self recovery to $0 \%$. Insets are schematic diagram of the device under $100 \%$ and $0 \%$ strain, respectively.

The sample with initial reflection wavelength at $640 \mathrm{~nm}$ was stretched to $100 \%$, and then the sample was released to recover to its initial state. Meanwhile, the change of light intensity at $640 \mathrm{~nm}$ was monitored with a spectrometer. The response time of optical signal and electrical signal can be regarded as consistent, because we think that the weak time difference is caused by different testing instruments. 


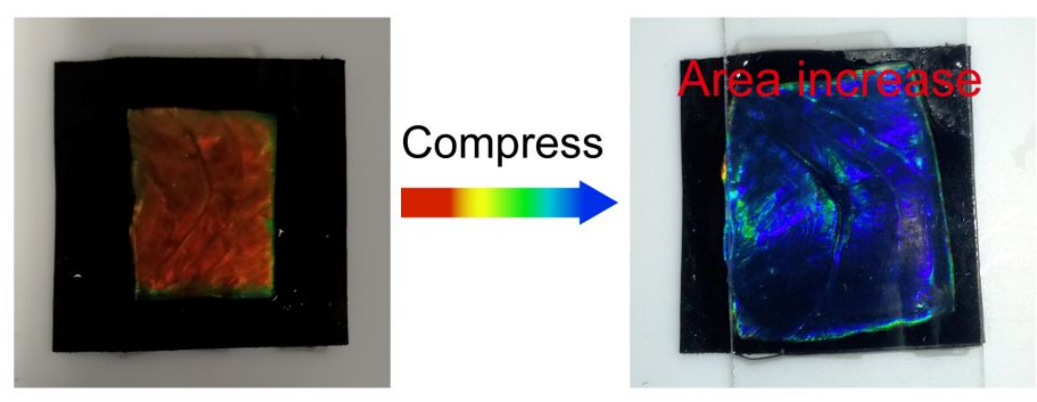

Figure S15. Area change of ICPH during compression. 


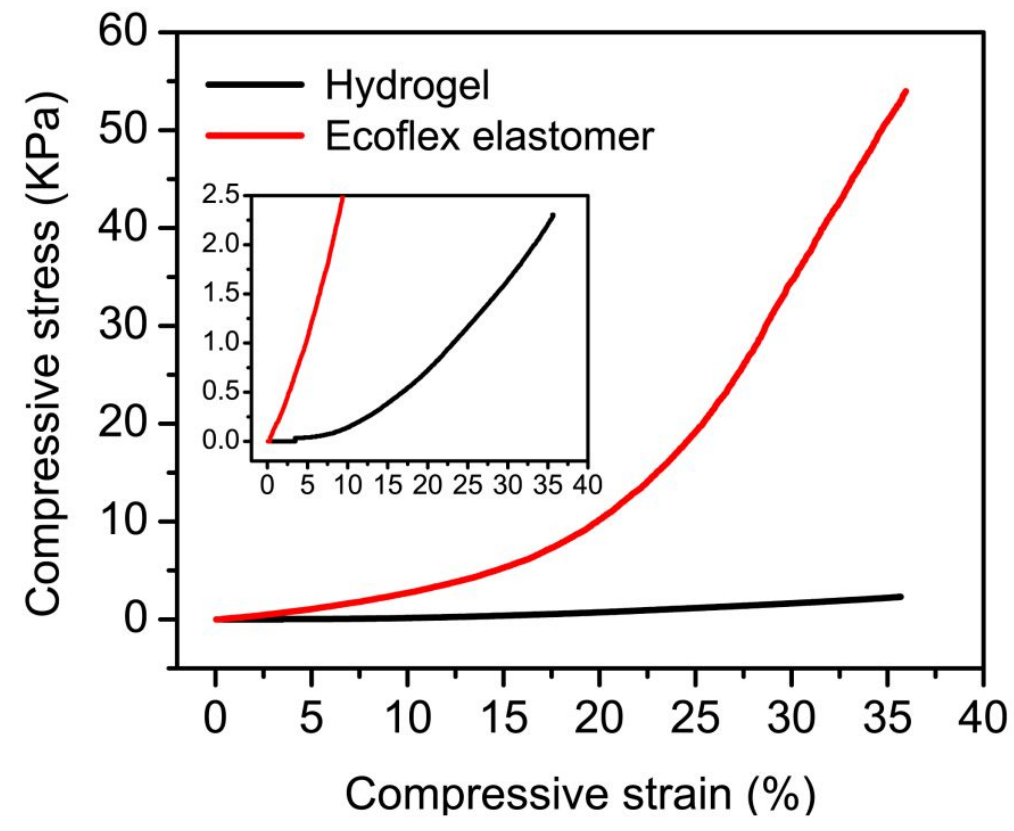

Figure S16. Compression stress-strain curves of ICPH and dielectric elastomer. Inset is a partial enlargement of the compressive strain from $0 \%$ to $35 \%$. 

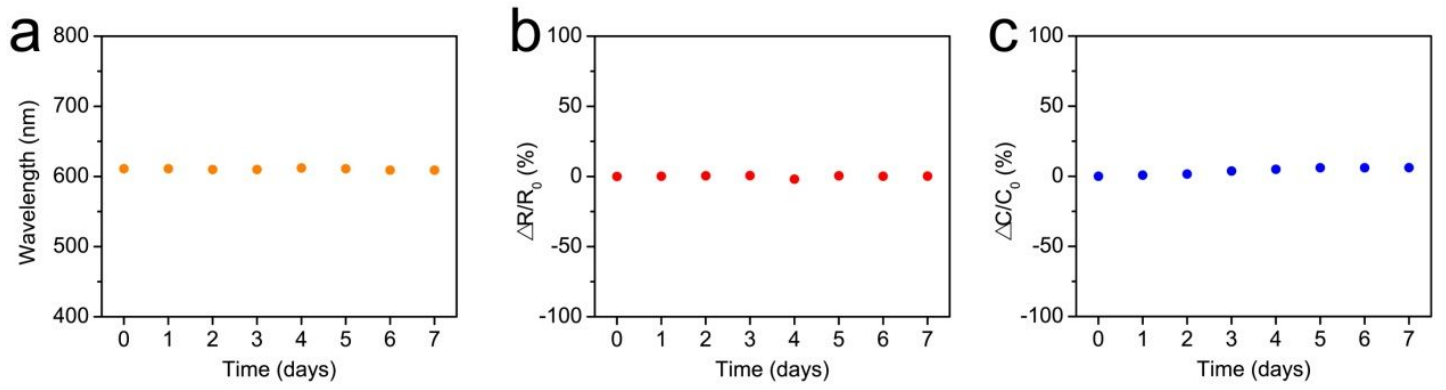

Figure S17. Stability of ionic sensory system encapsulated by epidermis-like VHB. (a)

Reflection wavelength change, (b) relative resistance change and (c) relative capacitance change of ionic sensory system. 

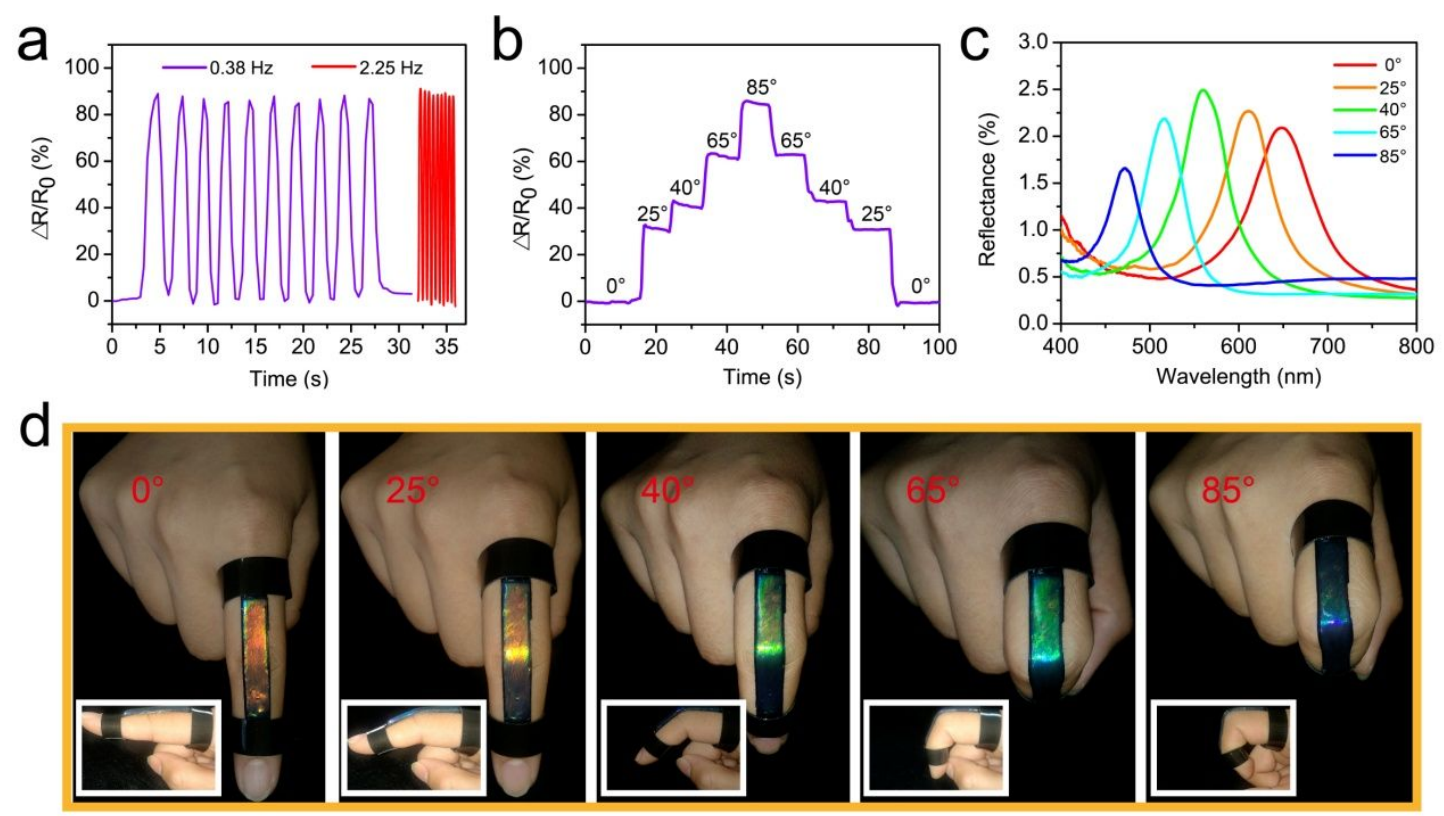

Figure S18. Responses of the biomimetic CIskin to human finger bending behavior.

(a) Changes of relative resistance with time to monitor the finger bending behavior in real time. (b) Changes of relative resistance under different bending degree of finger.

(c) Reflection spectrum of the device under different bending degree of finger. (d) Structural color under different bending degrees of finger. Insets reflect the bending degree of finger. 

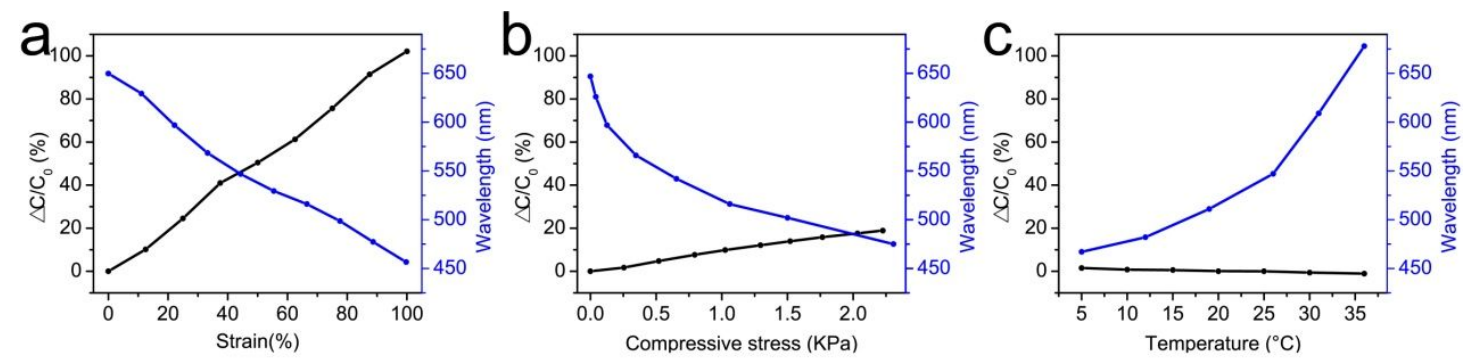

Figure S19. Changes in relative capacitance and reflection wavelength with (a) strain,

(b) compressive stress and (c) temperature.

The structural color of CIskin device changes after subjecting to different external stimuli. When the color changes are the same, the relative changes of capacitance signal caused by different external stimuli are different. When the sensor is stretched, the thickness of the dielectric layer $(d)$ decreases while the overlap area of two ionic hydrogels $(S)$ increases. ${ }^{\mathrm{S} 5}$ When the sensor is compressed, $d$ is almost unchanged and $S$ increases. In contrast, $d$ remains constant and the change of $S$ is negligible under different temperatures. Therefore, the capacitance increased rapidly, slowly, and negligibly, respectively, when the color changes are the same. As a consequence, we can distinguish different external stimuli through comparing the relative changes of the above two signals. 


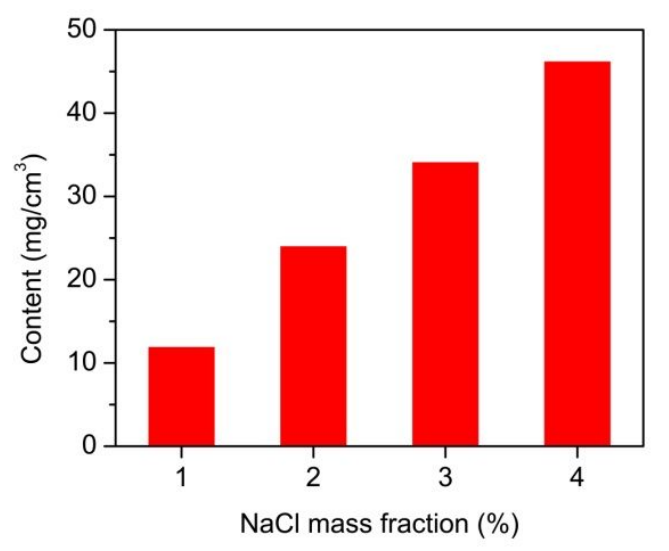

Figure S20. $\mathrm{NaCl}$ content in ICPH soaked in $\mathrm{NaCl}$ solution.

The hydrogels $(4 \mathrm{~cm} \times 2 \mathrm{~cm} \times 0.21 \mathrm{~cm})$ were immersed in $\mathrm{NaCl}$ solution $(1 \mathrm{wt} \%, 2$ $\mathrm{wt} \%, 3 \mathrm{wt} \%, 4 \mathrm{wt} \%)$ for $24 \mathrm{~h}$, and then annealed in muffle furnace $\left(600^{\circ} \mathrm{C}\right)$ for $3 \mathrm{~h}$ to to remove the organic matter and water. The residue was disposed in $25 \mathrm{~mL}$ solution, and the $\mathrm{Cl}^{-}$content was measured by ion chromatography to obtain the $\mathrm{NaCl}$ content ( $\mathrm{NaCl}$ mass/hydrogel volume). 


\section{Movies S1-S8}

Movie S1. Movie shows the dynamic color change of red ICPH during compression.

Movie S2. Movie shows that ICPH change from blue to red within $\sim 0.1 \mathrm{~s}$ at a large temperature difference, where a ICPH appears blue color in cold water at $\sim 8{ }^{\circ} \mathrm{C}$, and is heated by hot water $\left(\sim 45^{\circ} \mathrm{C}\right)$.

Movie S3, S4. Movies show a reversible anisotropic volume change behavior of ICPH under an optical microscope. When heated, the D increases, the L and W decrease, and its size returns to the initial state after cooling.

Movie S5. Movie demonstrates that the volume of pure PDAAM-co-PAAM matrix (without $\mathrm{C}_{12} \mathrm{DMAO}$ bilayers) does not change during heating/cooling.

Movie S6. Movie displays dynamic color change of the biomimetic skin during finger bending of the prosthesis.

Movie S7. Movie shows dynamic color switching of the biomimetic skin during finger bending of the human.

Movie S8. Movie indicates a visual response of blue ICPH to IR light, and the irradiated area quickly turns red due to heating. 


\section{Supplemental References}

S1. Platz, G.; Thunig, C.; Hoffmann, H. Iridescent Phases in Aminoxide Surfactant Solutions. Progr. Colloid Polym. Sci. 1990, 83, 167-175.

S2. Haque, M. A.; Kamita, G.; Kurokawa, T.; Tsujii, K.; Gong, J. P. Unidirectional Alignment of Lamellar Bilayer in Hydrogel: One-Dimensional Swelling, Anisotropic Modulus, and Stress/Strain Tunable Structural Color. Adv. Mater. 2010, 22, $5110-5114$

S3. Haque, M. A.; Kurokawa, T.; Kamita, G.; Gong, J. P. Lamellar Bilayers as

Reversible Sacrificial Bonds to Toughen Hydrogel: Hysteresis, Self-Recovery, Fatigue Resistance, and Crack Blunting. Macromolecules 2011, 44, 8916-8924.

S4. Wirthl, D.; Pichler, R.; Drack, M.; Kettlguber, G.; Moser, R.; Gerstmayr, R.; Hartmann, F.; Bradt, E.; Kaltseis, R.; Siket, C. M.; Schausberger, S. E.; Hild, S.; Bauer, S.; Kaltenbrunner, M., Instant Tough Bonding of Hydrogels for Soft Machines and Electronics. Sci. Adv. 2017, 3, e1700053.

S5. Sun, J. Y.; Keplinger, C.; Whitesides, G. M.; Suo, Z. Ionic Skin. Adv. Mater. 2014, $26,7608-7614$. 